
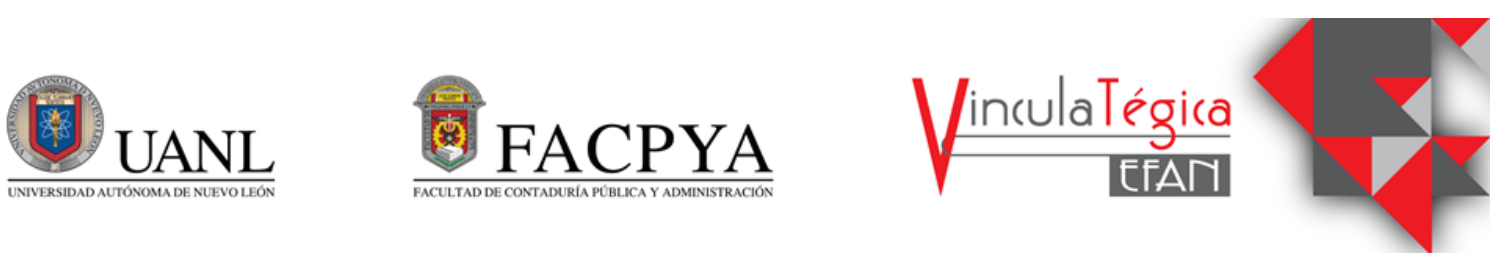

\title{
Instrumento de investigación validado para medir efectividad de redes sociales en época Covid-19
}

\author{
Mauricio Herrera Rodríguez ${ }^{1}$, Laura Elena Aguilar Herrera² y Manuel Eduardo Gutiérrez \\ Ortiz $^{3}$ \\ ${ }^{1}$ Universidad Autónoma de Tamaulipas, Facultad de Comercio y Administración de Tampico, Tampico, \\ Tamaulipas, México, mauherrera@docentes.uat.edu.mx, FCAT - UAT Campus Tampico S/N, (+52) \\ 8331553166 \\ ${ }^{2}$ Universidad Autónoma de Tamaulipas, Facultad de Comercio y Administración de Tampico, Tampico, \\ Tamaulipas, México, aguilarherrera@gmail.com, FCAT - UAT Campus Tampico S/N, (+52) 8331002774 \\ ${ }^{3}$ Universidad Autónoma de Tamaulipas, Facultad de Comercio y Administración de Tampico, Tampico, \\ Tamaulipas, México,mgutierrez@docentes.uat.edu.mx, FCAT - UAT Campus Tampico S/N, (+52)
} 8333007693

Información del artículo revisado por pares

Fecha de aceptación: junio-2021

Fecha de publicación en línea: diciembre-2021

DOI: https://doi.org/10.29105/vtga7.1-162

\section{Resumen}

Ante el advenimiento de la pandemia, las empresas y organizaciones tienen el reto de implementar nuevas habilidades para mantenerse competitivos y sustentables en un entorno que sufre cambios acelerados. La principal contribución que hace este artículo al conocimiento es aportar un instrumento de investigación del tema del uso de las redes sociales debido este desafío en el que surge la problemática de identificar si las redes sociales son un factor de productividad y desempeño. El objetivo de esta investigación fue definir un instrumento de investigación que permita medir la efectividad de las redes sociales en las PYMES en época de COVID19. La aportación práctica puede ayudar a los investigadores a contar con una herramienta que les apoye en sus investigaciones, además de sumar al estado del arte. La metodología consistió en una revisión documental de las teorías y seminales de redes sociales además de los estudios empíricos más recientes del tema para conformar cuatro cuestionarios de 10 preguntas cada uno que fue sometido a una prueba piloto de 80 sujetos similares a los de la población de estudio y después mediante un análisis factorial con una reducción de dimensiones con las pruebas de Bartlett y

\begin{abstract}
Faced with the advent of the pandemic, companies and organizations are challenged to implement new skills to stay competitive and sustainable in an environment that undergoes accelerated changes. Faced with this challenge, the problem arises of identifying whether social networks are a factor of productivity and performance. The objective of this research was to define a research instrument that allows to measure the effectiveness of social networks in SMEs in the time of COVID19. The practical contribution can help researchers to have a tool that supports them in their research, in addition to adding to the state of the art. The methodology consisted of a documentary review of the theories and seminars of social networks in addition to the most recent empirical studies of the subject to form four questionnaires of 10 questions each one that was subjected to a pilot test of 80 subjects similar to those of the population of study and then by means of a factorial analysis with a reduction of dimensions with the Bartlett and KMO tests, plus the determination of Cronbach's Alpha to define the items that make up the final research instrument. The results showed validity and
\end{abstract}


KMO, más la determinación del Alfa de Cronbach definir los ítems que conforman el instrumento de investigación final. Los resultados mostraron una validez y confiabilidad para el uso del instrumento al mismo tiempo que la determinación de nuevos constructos aplicables a las redes sociales.

Palabras clave: COVID19, Publicidad, PYMES, Redes sociales.

\section{INTRODUCCIÓN}

Las redes sociales se han convertido en algo esencial en los negocios y en las empresas, en particular para ofertar sus productos $y$ servicios. Shuting, Greenwood y Pavlou (2020) estiman que 8 de cada 10 pequeñas empresas, y el 99\% de las grandes empresas, han establecido páginas de redes sociales. Tan solo si se considera el reporte de Kemp (2018) El nuevo conjunto de informes Global Digital 2018 de We Are Social y Hootsuite revela que ahora hay más de 4 mil millones de personas en todo el mundo que utilizan Internet. Esto puede ser una gran oportunidad para las PYMES que se unan a esta ola tecnológica o una posible calamidad para quienes la ignoren.

Entre los trabajos previos que se han realizado sobre el tema se puede mencionar a Valsesia, Proserpio y Nunes (2020) ellos mencionan que los especialistas en marketing suelen generar información sobre productos y marcas a través de personas que se consideran influyentes en las redes sociales (influencers) y que esto tiene un efecto positivo en la influencia percibida. Por el contrario, Goldstein (2014) afirma que, aunque existe un fuerte deseo de las empresas de aprovechar las redes sociales, existen preocupaciones sobre los posibles efectos negativos del uso excesivo de las mismas ya que la publicación repetitiva de mensajes puede molestar a los seguidores y llevarlos a dejar de seguir a la empresa.

Además, la pandemia denominada COVID19 ha dejado formas diferentes de trabajar en las empresas y negocios. Barbolla (2020) comenta que se puede verificar cómo la pandemia COVID19 ha precipitado de manera importante el establecer y usar nuevas tecnologías de administración empresarial y él ha identificado los recursos digitales que reliability for the use of the instrument at the same time as the determination of new constructs applicable to social networks.

Key words: COVID19, Advertising, SMEs, Social Media

JEL Codes: M15, O33

tienen un mayor efecto sobre la productividad, entre las cuales se encuentra como una tendencia el uso de las redes sociales que llegan incluso a las operaciones de compras y ventas por medio de los apoyos tecnológicos.

Lo anterior da relevancia al objetivo de este estudio porque puede ser importante contar con instrumentos de investigación válidos y confiables que nos permitan medir, bajo un contexto científico y cuantitativo, la eficacia de las redes sociales, en particular en las PYMES. Como parte de la metodología que se desglosa más adelante se contempla una prueba piloto aplicada a más de 80 dueños, gerentes, jefes o encargados de los negocios de la zona comercial del centro de la ciudad de Tampico, Tamaulipas, en un estudio transversal realizado en febrero de 2021.

La hipótesis que se plantea es que el instrumento de investigación propuesto es válido y confiable para medir la variable Efectividad de las redes sociales en las PYMES por lo que el objetivo general de este trabajo es analizar los resultados de la aplicación de la prueba piloto y su posterior análisis estadístico para determinar que son robustos para contar con dicho instrumento de investigación para investigaciones futuras y definir un constructo basado no solo en la literatura, sino también en un estudio empírico.

\subsection{Planteamiento del problema}

Al revisar artículos acerca de las redes sociales como los de Curmi, Said y Attard (2019) que estudian como estas han influido en varias áreas incluso las de la porlitica o como Qiu, Tang y Whinston (2015) que analizan dos fórmulas para el éxito en las redes sociales que son el aprendizaje y los efectos de red, más Assegaff et al. (2017) que validan un 
modelo de éxito en redes sociales para compartir conocimientos y Degand (2015) que realiza un estudio fenomenológico de varios casos sobre habilidades sociales, aspiraciones y experiencias relacionadas con los medios de comunicación. Esto determina lo complicado que puede resultar medir la efectividad de las redes sociales en las PYMES.

La era digital no solo ha alterado el comportamiento de los consumidores, sino que también ha hecho que la mercadotecnia en redes sociales sea extremadamente compleja. La televisión y la radio ahora trabajan junto con la mercadotecnia en redes sociales digital para llegar y atraer a los consumidores. Si los especialistas en mercadotecnia en redes sociales no pueden obtener una visión integral de los clientes mientras atraviesan múltiples puntos de contacto y dispositivos, entonces no pueden medir con precisión el valor de cada experiencia o qué tan bien los están moviendo hacia sus criterios de éxito deseados. (Anderson, Wolff, 2010) por lo que el contenido atractivo resulta importante para la efectividad de las redes sociales en las PYMES.

Jung, Dieck y Chung (2018) comentan que con la aparición de los dispositivos móviles los factores como la interacción y la propagación se han vuelto cada vez más importantes en el uso de las redes sociales. Sin embargo, con respecto al uso de las redes sociales por parte de los clientes, el conocimiento sobre cómo esos factores contribuyen al uso continuo y consumo sigue siendo limitado, de ahí la importancia de examinar la influencia de la interacción y constancia en las publicaciones con la confianza, los beneficios y el disfrute del uso continuo de los productos o servicios de las empresas, es decir la eficacia de las redes sociales puede depender de que las publicaciones en las mismas sean continuas.

Aladwani (2017) afirma que crear y mantener relaciones favorables en las redes sociales con los clientes requiere que las organizaciones produzcan contenido de calidad que se adapte a las necesidades de los clientes. En una muestra de usuarios activos de redes sociales se revelan al menos tres hallazgos dignos de mención. En primer lugar, el estudio confirmó la multidimensionalidad de la calidad compatible del contenido de las redes sociales, que abarca componentes reflexivos, estimulados, practicados y defendidos. En segundo lugar, hubo equivalencia en las mediciones de los constructos estudiados y los pesos estructurales de la red de relaciones propuesta entre los diferentes subgrupos de género y experiencia. En tercer lugar, se descubrió que la calidad compatible del contenido de las redes sociales influye en el interés continuo, la confianza activa y la apertura a la retroalimentación. Por lo tanto, medir la calidad de los mensajes puede también ser un factor que impacte en la efectividad de las redes sociales de las PYMES.

De todo lo anterior se puede concluir que, a pesar de existir extensa literatura sobre redes sociales, no se descubrió en las bases de datos consultadas como EBSCO, ELSEVIER y WILEY, documentos que relacionen a la efectividad de las redes sociales con los conceptos de contenido atractivo, continuidad de las publicaciones, así como la calidad de estas. Por lo que el planteamiento del problema es que no existe un instrumento de investigación que relacione a la efectividad las redes sociales de los negocios PYMES con estos conceptos.

\subsection{Pregunta de investigación}

Las preguntas propuestas de esta investigación que parten de una investigación documental de revistas indexadas tipo JCR o SCOPUS. ¿Son las adecuadas para soportar el constructo de efectividad de redes sociales en las PYMES?

\subsection{Objetivo general}

Determinar mediante pruebas estadísticas si las preguntas propuestas basadas en estudios empíricos que se muestran en este artículo respaldan un instrumento de investigación validado y confiable.

\subsection{Proposiciones}

Las preguntas formuladas a partir de estudios empíricos que se muestran en este artículo respaldan un instrumento de investigación validado y confiable. 


\section{MARCO TEÓRICO}

\subsection{Teorías seminales}

La Teoría Funcionalista es desarrollada de manera importante por el sociólogo Talcott Parsons con sus obras Un esbozo del sistema social, Estructura y proceso en las sociedades modernas y la Estructura de la Acción (Parsons, 1961; 1967 y 1971) que más adelante es integrada por Rendón (2007) en su libro Teoría e investigación de la comunicación de masas, más adelante por Vélez, G. (2008) en su artículo Exploración de las relaciones entre redes sociales y comunicación así como por Sasín, (2018) con su estudio La comunicación de masas que complementa todo con la teoría de Luhmann (2000).

La teoría de las redes sociales vincula las teorías anteriores, funcionalista y de comunicación de masas, con la teoría de Barnes (1954) que introduce la importancia de la amistad, de las relaciones familiares e incluso las de vecindad. Así como, Bott (1955) con el énfasis en el parentesco, que es reforzado por Barnes y Harary (1983) quienes le dan un sentido teórico a las redes sociales y realizan estudios con métodos científicos con los que llegan a la idea de que las relaciones sociales son redes que tienen repercusiones en modelos aplicables a la vida real por último, aunque sin agotar el tema de los artículos seminales se puede mencionar a Galaskiewicz y Wasserman (1993) que introduce los temas de círculos sociales y unión entre los individuos.

\subsection{Estudios empíricos relacionados}

\subsubsection{Redes sociales}

Siguiendo el paradigma de Oliver (1980) de las decisiones basadas en el modelo cognitivo de los antecedentes y consecuencias de la satisfacción, se puede estimar que los efectos de red para el consumo de contenido de redes sociales pueden dar una experiencia positiva.

Esto ocurre al identificar la desconfianza o sorpresa que resulta de comparar las expectativas previas, del espectador, con las reales y de acuerdo con este paradigma, la expectativa es la referencia que tiene dicho espectador y que puede ser negativa o positiva y se califica de abajo hacia arriba, es decir que cuando dicho espectador selecciona videos en línea, genera un éxito en las redes sociales que repercuten en las elecciones comunes que los consumidores hacen todos los días en la vida real. (Qiu, Tang, y Whinston, (2015) lo que es bueno para las PYMES.

Por su parte Nonaka (2008) afirma que, al utilizar las redes sociales, las personas tienen la oportunidad de desarrollar su red y sus comunidades lo les ayudarían a compartir experiencias y conocimientos. Además de que el intercambio de conocimientos es una de las claves para crear seres que tengan las habilidades y competencias confiables. Este autor en su estudio afirmó que el intercambio de conocimientos solo puede llegar a través de actividades de interacción y colaboración que se dan en las redes sociales.

También, DeLone y McLean (2002) crearon un modelo para medir el éxito de los sistemas de información en el que midieron 6 elementos que son la calidad, la información, los servicios, el nivel de uso, la satisfacción del usuario y el beneficio. Este modelo fue muy exitoso en su ejecución con los sistemas de información que fue también adaptado al comercio electrónico que dio pie a otro estudio exitoso, denominado Midiendo el éxito del comercio electrónico: Aplicación del modelo de éxito de los sistemas de información. (DeLone y McLean, 2004) lo que relaciona las redes sociales con las ventas porque como afirma Kotler (2002) toda venta sigue un proceso conformado por varias etapas denominado proceso de ventas, que va desde la prospección o búsqueda de clientes potenciales hasta el cierre de la venta y la posterior relación de postventa.

Por otra parte, en cualquier organización, el departamento de ventas juega un papel fundamental en el éxito del negocio. $Y$ el intercambio es el acto mediante el cual se obtiene un objeto dado, perteneciente a otra persona, ofreciéndole algo a cambio. Si este se realiza de manera más efectiva le correlación con el éxito de las PYMES debería ser significativa. (Kotlery Amstrong, 2004)

Por lo anterior se define para este 
estudio la definición de Efectividad del uso de redes sociales al incremento de ventas, la mejora en la imagen del negocio y el aumento de clientes en las empresas PYMES.

\subsubsection{Contenido atractivo}

El reporte del boletín de Delta Kappa Gamma (2017) afirma que el tema de las redes sociales es enorme y, a veces, abrumador porque las redes sociales se han convertido en parte de la vida cotidiana de personas de todas las edades. Millones de personas utilizan las redes sociales para informarse y compartir intereses. El artículo analiza diferentes tipos de redes sociales y cómo se utilizan para comunicaciones y publicidad. Los temas incluyen los tipos de redes sociales, incluidas las redes sociales, las redes comerciales y las redes sociales por grupos de interés, las pautas para el uso seguro de las redes sociales y la introducción a varias redes sociales, como Pinterest, Twitter y el blog de comunicaciones y publicidad donde lo que se destaca como un común denominador es que el contenido de las publicaciones en las redes sociales sea atractivo.

También Zahid et al. (2018) comentan que, con la ayuda de nuevas herramientas de comunicación como las redes sociales, la motivación detrás del comportamiento de los consumidores hacia los productos es diferente en comparación con otros productos en general. $\mathrm{Al}$ explorar los factores que determinan la intención de compra de productos y la publicidad en las redes sociales. Esto abarca múltiples aspectos que van desde lo ecológico hasta lo económico pero un detalle importante es que la publicidad atractiva es relevante para la toma de decisiones de los consumidores.

Larimo y Leonidou (2021) encuentran cuatro formas de estrategias de marketing en las redes sociales que son la estrategia de comercio social, contenido social, monitoreo social y una cadena de valor para el consumidor social, ellos denotan niveles progresivos de madurez estratégica, y que esto está determinado por los objetivos estratégicos, los recursos y las capacidades de la empresa. Sin embargo, el éxito de estas estrategias dependerá de la capacidad de la empresa para identificar y aprovechar lo que es atractivo para el cliente, como en el caso de transformar a los clientes de receptores pasivos de las ofertas de redes sociales de la empresa en contribuyentes activos de valor que compran los productos. Lo que repercute en la importancia de que las publicaciones sean atractivas.

En este estudio se define al contenido atractivo como las publicaciones que se hacen en redes sociales que identifican, aprovechan lo que es interesante para aumentar la intención de compra de los consumidores.

\subsubsection{Continuidad de las publicaciones}

Sweeney y col. (2012) hace referencia a que las publicaciones deben tener continuidad porque la comunicación informal, de lo que se puede llamar boca a boca virtual, influye en la evaluación y compra de productos y servicios. Esto por supuesto se vuelve relevante cuando las redes sociales, tanto que algunas empresas están comenzando a recortar presupuestos en otros esquemas de publicidad tradicional para llevarlos a las redes sociales y buscar que sean Temas Trascendentes (TT) lo que se conoce en inglés como Trending Topic, que es una forma de destacar y clasificas los términos más utilizados en la plataforma Twitter y que se ha generalizado a otras redes sociales, donde la comunicación boca a boca virtual se vuelve relevante para la efectividad de las redes sociales.

Por su parte Finsterwalder y col. (2011) coinciden en este fenómeno de los TT y ellos relacionan a la comunicación con la satisfacción y la calidad del servicio. En su estudio ellos plantean que los clientes satisfechos comparten sus experiencias con otros cinco clientes, mientras que los clientes insatisfechos comparten sus experiencias con otros 15 clientes. Por lo tanto, los especialistas en mercadotecnia y publicidad en redes sociales buscan enfatizar la retroalimentación de los clientes cuando se trata de la calidad del servicio positiva porque esa comunicación de boca a boca virtual es más efectiva y económica además de que los clientes potenciales usarán el boca a boca para difundir noticias sobre los productos y servicios de las 
compañías.

Fong y Burton (2006) encontraron algo similar en el que la opinión de las personas afines y las recomendaciones sobre cualquier producto o servicio son de suma importancia. Por lo que la continuidad en los mensajes por parte de las PYMES puede ser importante, máximo en un ambiente en el que los medios digitales permiten a los consumidores tener diversas personas afines en distintas partes del mundo.

Por último, Andreassen y Streukens (2009) afirman que el boca a boca virtual puede ser más contundente cuando los comentarios surgen de personas que no son parte de la compañía, es decir otros consumidores o clientes y que por lo tanto no tienen objetivos comerciales al expresar sus ideas acerca de productos y servicios. Esto hace relevante que la continuidad en las publicaciones sea importante para la efectividad de estas.

Por lo anterior se define en esta investigación a la continuidad de las publicaciones como la frecuencia de textos, imágenes o videos que se publican en las redes sociales y que permiten incrementar el boca a boca virtual para difundir información acerca de productos o servicios de una empresa.

\subsubsection{Calidad de las publicaciones}

Cho (2015) define que en la competencia de negocios que ofrecen sus productos y servicios se ha intensificado de manera importante por lo que las empresas y en particular las PYMES deben cuidar la calidad en el servicio que prestan por medio de internet por lo que esta participación en las redes sociales un papel fundamental en la identificación de PYMES confiables. Parasuraman, Zeithaml y Malhotra (2005).: diseñaron una escala de varios elementos para evaluar la calidad del servicio electrónico denominada ES-QUAL que ha validado que la calidad del servicio electrónico influye en la satisfacción y la lealtad del cliente por lo que es importante contemplarla en el uso de las redes sociales.

Parasuraman, Zeithaml y Berry (1985) ya habían definido un modelo conceptual de la calidad del servicio y sus implicaciones para futuras investigaciones, esto en época de COVID19 es más importante ya que Internet mejora significativamente la simetría de información entre vendedores y compradores donde las reseñas de los clientes en línea, incluidas las redes sociales, contribuyen en gran medida a mejorar las opiniones.

Jakic, Wagner, y Meyer (2017). Estudiaron el impacto de la adaptación del estilo del lenguaje durante las interacciones en las redes sociales en la confianza de la marca y confirman lo evidente de que los clientes pueden publicar comentarios sobre su experiencia de compra a través de plataformas sociales en línea como Facebook, Twitter o YouTube y que esa información se comparte con una gran cantidad de usuarios de redes sociales lo que potencialmente influye en el comportamiento de otros compradores online. Es decir, en esta era de Internet, la información que indica la calidad del servicio de las PYMES se ha vuelto más crítica porque los compradores en línea utilizan esta información para seleccionar donde comprar.

Por lo anterior en esta investigación se define a la calidad de las publicaciones en redes sociales como la satisfacción de los usuarios de redes sociales que ven los anuncios y encuentran simetría que los lleva a comprar productos o servicios.

\section{MÉTODO}

Esta es una investigación no experimental, cuantitativa, de carácter exploratorio, observacional y transversal. El objetivo es verificar la validez total del instrumento de investigación con el método que se describe a continuación.

\subsection{Diseño de los cuestionarios.}

El rigor analítico de este instrumento está basado en el estudio de las investigaciones documentales del estado del arte y con ello definir las variables que pueden intervenir en la eficacia del uso de las redes sociales mediante una técnica documental en la que se revisaron diversas bases de datos de artículos de revistas indexadas JCR o SCOPUS. Se definió un cuestionario de 10 preguntas para cada variable, tipo Likert de cinco niveles con opciones de nada, poco, más o menos, mucho 
y totalmente, con lo que se conforma un instrumento de investigación. En seguida se aplicó dicho instrumento a 80 sujetos de investigación y se realizó una reducción de dimensiones con rotación VARIMAX para definir los ítems validados a los cuales se les aplicó un analisis de confiabilidad con el Alfa de Cronbach, buscando que los valores fueran mayores de 0.7 coincidiendo con Tuapanta, Duque y Mena (2017)

Los cuestionarios iniciales quedan conformados con las siguientes preguntas que se presentan en la tabla 1 y tabla 2 .

\subsection{Modelo conceptual.}

A partir de las variables definidas en el marco teórico el modelo conceptual que definido como se muestra en la figura 1 .

\subsection{Población, muestra y sujetos de estudio.}

La población de estudio corresponde a los negocios PYMES como los identifican Cardozo, de Naime y Monroy (2012) con criterios de tamaño del negocio de locales con hasta tres oficinas o habitaciones y con un número de empleados menor de 50 empleados. Al ser una prueba piloto la muestra se realizó por conveniencia en la zona comercial del primer cuadro de la ciudad de Tampico, Tamaulipas que incluye 20 manzanas o cuadras y los sujetos de estudio fueron los dueños, gerentes, jefes o encargados de los negocios contactados vía telefónica a los cuales se les pidió que contestaran los cuestionarios montados en la plataforma Google Forms.

\section{RESULTADOS}

Después de aplicar los cuestionarios con rigor crítico en la prueba piloto se realizó para cada uno de los mismos un análisis factorial para buscar una reducción de dimensiones con rotación VARIMAX y se les aplicó la prueba de Bartlett y la KMO, lo que le da rigor de contenido dando los datos mostrados en la tabla 3.

De lo anterior los ítems validados y confiables corresponden a los elementos que alcanzaron un alfa de Cronbach superior a 0.7 y se presentan en las tablas 4,56 y 7 .

\section{CONCLUSIONES}

$\mathrm{Al}$ analizar los resultados se puede deliberar o discutir de manera precisa que, en cada variable de las 10 preguntas originales, basadas en la investigación documental ya descrita, se redujeron a pocos componentes, cada uno con una cantidad de preguntas específica. En todas las variables la medida Kaiser - Meyer - Olkin de adecuación de muestreo da valores adecuados y la prueba de esfericidad de Bartlett también es significativa. Además, la varianza explicada acumulada muestra una buena representación de lo que se desea medir con lo que se concluye que el instrumento de investigación resultante cuenta con validez.

Sin embargo, al calcular el Alfa de Cronbach de cada uno de los componentes solo algunos de los elementos dan valores aceptable mayores de 0.7 por lo que se eliminan diversas preguntas que dejan paso a los ítems del instrumento de investigación validado y confiable y que permite definir los constructos siguientes basados en un estudio empírico, cuantitativo y con rigor científico.

Eficacia del uso de redes sociales se define como el aumento de la imagen de la empresa que genera más utilidades, satisfacción de los clientes y muestra un incremento en los indicadores de "me gusta" en las publicaciones de las redes sociales de la empresa.

El contenido atractivo se define como la identidad de los usuarios con la publicidad que muestra la empresa cuando usa contenido audiovisual colorido, con colores vividos en videos y mediante varias plataformas que se reflejan en los comentarios en las redes sociales.

Continuidad de las publicaciones se define como el publicar 2 o más publicidades por semana que generen comentarios, opiniones, retroalimentación y comunicación de boca a boca virtual con la empresa.

Calidad de las publicaciones se define como la simetría que genera la empresa con los clientes y que provoca la confianza, lealtad, expresiones de satisfacción porque la publicidad contiene información con los datos de los productos y un buen servicio al cliente 
después de mostrar las publicaciones.

En tiempos de la pandemia de COVID19 con las restricciones laborales y comerciales en 2020 y 2021, las empresas requieren de nuevas formas de promover sus negocios y comercializar sus productos o servicios. Por si mismos estos constructos permiten inferir las acciones que pueden realizar las empresas PYMES para mejorar el uso de sus redes sociales y los posibles beneficios que se obtendrían. Queda, sin embargo, para investigaciones futuras el realizar estudios de correlación que generen algún modelo predictivo utilizando una muestra mayor. 


\section{REFERENCIAS}

Aladwani, A. M. (2017). Compatible quality of social media content: Conceptualization, measurement, and affordances. International Journal of Information Management, 37(6), 576-582.

Anderson, C., \& Wolff, M. (2010). The Web is dead. Long live the Internet. Wired Magazine, 18(15), 1-12.

Andreassen, T. W., \& Streukens, S. (2009). Service innovation and electronic word-of-mouth: is it worth listening to?. Managing Service Quality: An International Journal.

Assegaff, S., Hendri, Sunoto, A., Yani, H., \& Kisbiyanti, D. (2017). Social Media Success Model for Knowledge Sharing (Scale Development and Validation). Telkomnika, 15(3), 13351343.

Barbolla, J. O. (2020). Transformación digital, redes sociales y comercio electrónico en la estrategia empresarial frente a la covid-19. Actividad empresarial en la pandemia de la covid-19 $\mathrm{N}^{\mathrm{o}} 170,140$.

Barnes J.A., Harary F. (1983) 'Graph Theory in Neetwork Aanlysis' en Social Networks, 5.

Barnes, J. A. (1954). Class and committees in a Norwegian island parish. Human relations, 7(1), 39-58.

Bott E. (1955) 'Urban Families: Conjugal Roles and Social Networks' en, Humans Relations, 8.

Cardozo, E., de Naime, Y. V., \& Monroy, C. R. (2012, May). El concepto y la clasificación de PYME en América Latina. In Global Conference on Business and Finance Proceedings (Vol. 7, No. 2, pp. 1657-1668).

Cho, Y. K. (2015). Creating customer repurchase intention in Internet retailing: The effects of multiple service events and product type. Journal of Retailing and Consumer Services, 22, 213-222.

Communications \& Publicity Connecting with Social Media: Personally, Professionally, and Educationally. (2017). Delta Kappa Gamma Bulletin, 83(4), 57-59.

Curmi, F., Said, E., \& Attard, C. (2019). Political Campaign Success Predictors from Social Media Financing. Journal of Management Policy \& Practice, 20(5), 101-110.

Degand, D. (2015). A Phenomenological Multi-Case Study About Social Success Skills, Aspirations, and Related Media Experiences. Qualitative Report, 20(6), 872-900.

DeLone, W. H., \& McLean, E. R. (2002). Information systems success revisited. In Proceedings of the 35th Annual Hawaii International Conference on System Sciences (January pp. 29662976). IEEE.

DeLone, W. H., \& McLean, E. R. (2004). Measuring e-commerce success: Applying the DeLone \& McLean information systems success model. International Journal of electronic commerce, 9(1), 31-47.

Finsterwalder J, Garry T, Lang B. (2011). How word of mouth communication varies across service encounters. Managing Service Quality: An International Journal 21(6): 583-598.

Fong, J., \& Burton, S. (2006). Electronic word-of-mouth: a comparison of stated and revealed behavior on electronic discussion boards. Journal of Interactive Advertising, 6(2), 61-70.

Galaskiewicz, J., \& Wasserman, S. (1993). Social network analysis: Concepts, methodology, and directions for the 1990s. Sociological Methods \& Research, 22(1), 3-22.

Goldstein, D. G., Suri, S., McAfee, R. P., Ekstrand-Abueg, M., and Diaz, F. 2014. “The Economic and Cognitive Costs of Annoying Display Advertisements," Journal of Marketing Research (51:6), pp. 742-752.

Jakic, A., Wagner, M. O., \& Meyer, A. (2017). The impact of language style accommodation during social media interactions on brand trust. Journal of Service Management.

Jung, T. H., Dieck, M. C. T., \& Chung, N. (2018). Determinants of hotel social media continued usage. International Journal of Contemporary Hospitality Management.

Kemp, S. (2018). Digital in 2018: World's internet users pass the 4 billion mark. We are social, 30.

Li, F., Larimo, J., \& Leonidou, L. C. (2021). Social media marketing strategy: definition, 
conceptualization, taxonomy, validation, and future agenda. Journal of the Academy of Marketing Science, 49(1), 51-70.

Luhmann, Niklas (2000). La Realidad de los Medias de Masas, Anthropos, Barcelona.

Nonaka, I. (2008). The Knowledge-Creating Company (Harvard Business Review Classics). Harvard Business School Pr, ISBN.

Oliver, R.L. A (1980) Cognitive model of the antecedents and consequences of satisfaction decisions. Journal of Marketing Research, 17, 4, 460-469.

Parasuraman, A., Zeithaml, V. A., \& Berry, L. L. (1985). A conceptual model of service quality and its implications for future research. Journal of marketing, 49(4), 41-50.

Parasuraman, A., Zeithaml, V. A., \& Malhotra, A. (2005). ES-QUAL: A multiple-item scale for assessing electronic service quality. Journal of service research, 7(3), 213-233.

Parsons, T. (1961). An Outline of the Social System. In Theories of Society: Foundations of Modern Sociological Theory. Vol I (pp. 30-79). New York: The Free Press.

Parsons, T. (1967). La posición actual y las perspectivas de la teoría sistemática en Sociología. In Ensayos de Teoría Sociológica. Buenos Aires: Paidós.

Parsons, T. (1971). La Estructura de la Acción Social. Tomo 1. Madrid: Guadarrama

Qiu, L., Tang, Q., \& Whinston, A. B. (2015). Two Formulas for Success in Social Media: Learning and Network Effects. Journal of Management Information Systems, 32(4), 78-108.

Rendón, J. C. L. (2007). Teoría e investigación de la comunicación de masas. Pearson educación.

Sasín, M. G. (2018). La comunicación de masas. La semántica de lo masivo en la teoría de Niklas Luhmann. In X Jornadas de Sociología de la Universidad Nacional de La Plata (Ensenada, 5 al 7 de diciembre de 2018).

Shuting (Ada) vWang, Greenwood, B. N., \& Pavlou, P. A. (2020). Tempting Fate: Social Media Posts, Unfollowing, and Long-Term Sales. MIS Quarterly, 44(4), 1521-1571.

Sweeney JC, Soutar GN, Mazzarol T. (2012). Word of mouth: measuring the power of individual messages. European Journal of Marketing 46(1/2): 237-257

Tuapanta Dacto, Jorge Vinicio, Duque Vaca, Miguel Angel, Mena Reinoso, Angel Patricio (2017). Alfa de Cronbach para validar un Cuestionario de uso de TIC en Docentes Universitarios. Revista mktDescubre-ESPOCH FADE, (10), 37-48. Latindex Folio 20003, ISSN - 1390 7352 IEPI Título $\mathrm{N}^{\circ} 3232$ - 12

Valsesia, F., Proserpio, D., \& Nunes, J. C. (2020). The Positive Effect of Not Following Others on Social Media. Journal of Marketing Research (JMR), 57(6), 1152-1168.

Vélez, G. (2008). Exploración de las relaciones entre redes sociales y comunicación. Razón y palabra, (61).

Zahid, M. M., Ali, B., Ahmad, M. S., Thurasamy, R., \& Amin, N. (2018). Factors Affecting Purchase Intention and Social Media Publicity of Green Products: The Mediating Role of Concern for Consequences. Corporate Social Responsibility \& Environmental Management, 25(3), 225-236. 
FIGURAS

Figura 1. Modelo conceptual.

\begin{tabular}{|l|}
\hline \multicolumn{4}{|c|}{ Preguntas de variables } \\
- $\quad$ Efectividad del uso de \\
redes sociales \\
- Contenido atractivo \\
publicaciones \\
$\begin{array}{l}\text { Continuidad en las } \\
\text { publicaciones }\end{array}$ \\
\hline
\end{tabular}

\begin{tabular}{|l|l|}
$\begin{array}{l}\text { Prueba de Barlett y KMO } \\
\text { significativas }\end{array}$ & $\begin{array}{l}\text { Constructos } \\
\text { definidos con ítems } \\
\text { validados y } \\
\text { Valor del Alfa de Cronbach } \\
\text { superior a } 0.7\end{array}$ \\
\hline
\end{tabular}

Fuente: Elaboración propia

\section{TABLAS}

Tabla 1. Ítems propuestos para las variables parte 1

\begin{tabular}{ll}
\hline Variable Efectividad del uso de redes sociales & Variable contenido atractivo \\
\hline Aumento de ventas & Usuarios se identifican con las publicaciones \\
Aumento de la imagen del negocio & Se usan imágenes coloridas \\
Incremento de clientes & Uso audiovisual \\
Mayores utilidades & Videos interesantes \\
Disminución de mermas & Publicaciones que motivan comentarios \\
Mayor prestigio en el mercado & Cambio de usuarios a clientes \\
Comentarios positivos de clientes & Monitoreo del gusto de los usuarios de las redes \\
Incremento de ventas foráneas & Publicidad basada en emociones \\
Mayor satisfacción de clientes & Impacto de las publicaciones en la vida real \\
Incremento de Me Gusta en las redes sociales & Uso de diversas plataformas de redes sociales \\
\hline Fuente. Elaboración propia &
\end{tabular}

Tabla 2. Ítems propuestos para las variables parte 2

\begin{tabular}{ll}
\hline Variable Continuidad de las publicaciones & Variable Calidad de las publicaciones \\
\hline Más de 2 publicaciones por semana & Atención al servicio post publicidad \\
Mayores comentarios de las publicaciones & Percepción de confiabilidad en la publicidad \\
Comunicación bidireccional por publicidad & Mejora en la lealtad del cliente \\
Mayor comunicación boca a boca virtual & Simetría entre compradores y empresa \\
Experiencias compartidas por usuarios & Lenguaje inclusivo con los clientes \\
Retroalimentación con clientes & Información relevante de productos o servicios \\
Secuencia en la difusión de mensajes & Mejora en la confianza de la compañía \\
Identificación de personas afines extranjeras & Expresiones de satisfacción de la calidad \\
Opiniones de usuarios sin interés particular & Datos y detalles objetivos de los productos \\
Frecuencia al compartir ideas entre clientes & Incremento de compras por calidad \\
\hline
\end{tabular}

Fuente. Elaboración propia 
Tabla 3. Validez del instrumento por reducción de dimensiones

\begin{tabular}{|c|c|c|c|c|c|c|c|}
\hline Variable & $\begin{array}{l}\text { Preguntas } \\
\text { Originales }\end{array}$ & $\begin{array}{l}\text { Compo } \\
\text { nente }\end{array}$ & $\begin{array}{c}\text { Preguntas } \\
\text { Por } \\
\text { componente }\end{array}$ & KMO & Bartlett & $\begin{array}{l}\text { Varianza } \\
\text { explicada }\end{array}$ & $\begin{array}{l}\text { Alfa de } \\
\text { Cronbach }\end{array}$ \\
\hline \multirow{3}{*}{$\begin{array}{l}\text { Eficacia del uso } \\
\text { de redes sociales }\end{array}$} & 10 & 1 & 3 & 0.815 & 0.000 & 85.34 & 0.622 \\
\hline & & 2 & 4 & & & & 0.834 \\
\hline & & 3 & 3 & & & & 0.421 \\
\hline \multirow{2}{*}{$\begin{array}{l}\text { Contenido } \\
\text { atractivo }\end{array}$} & 10 & 1 & 6 & 0.846 & 0.000 & 73.45 & 0.893 \\
\hline & & 2 & 4 & & & & 0.642 \\
\hline \multirow{2}{*}{$\begin{array}{l}\text { Continuidad de } \\
\text { las publicaciones }\end{array}$} & 10 & 1 & 9 & 0.858 & 0.000 & 78.47 & 0.865 \\
\hline & & 2 & 1 & & & & 0.432 \\
\hline \multirow{2}{*}{$\begin{array}{l}\text { Calidad de las } \\
\text { publicaciones }\end{array}$} & 10 & 1 & 9 & 0.843 & 0.000 & 71.89 & 0.884 \\
\hline & & 2 & 1 & & & & 0.242 \\
\hline
\end{tabular}

Fuente. Elaboración propia

Tabla 4. Descriptivos de ítems del constructo Eficacia del uso de redes sociales

\begin{tabular}{lccc}
\hline Estadísticas de elemento & Media & Desviación estándar & $\mathrm{N}$ \\
\hline AumImagen & 2.35 & 1.032 & 85 \\
MasUtilidad & 2.29 & .986 & 85 \\
SatisfClientes & 2.12 & .878 & 85 \\
MasMeGusta & 2.33 & 1.016 & 85 \\
\hline
\end{tabular}

Fuente. Elaboración propia

Tabla 5. Descriptivos de ítems del constructo contenido atractivo

\begin{tabular}{lccc}
\hline Estadísticas de elemento & Media & Desviación estándar & $\mathrm{N}$ \\
\hline UsuarIdentPub & 2.80 & 1.121 & 85 \\
ImagenColor & 2.65 & .948 & 85 \\
Audiovisual & 2.67 & 1.073 & 85 \\
VideosInteres & 2.61 & 1.135 & 85 \\
PubComent & 2.78 & 1.138 & 85 \\
DiversPlataform & 2.87 & 1.153 & 85 \\
\hline Fuente. Elaboración propia & & &
\end{tabular}

Tabla 6. Descriptivos de ítems del constructo Continuidad de las publicaciones

\begin{tabular}{lccc}
\hline Estadísticas de elemento & Media & Desviación estándar & $\mathrm{N}$ \\
\hline DosOmasPub & 1.96 & .837 & 85 \\
MAsComent & 1.98 & .886 & 85 \\
ComunBidirec & 2.21 & 1.025 & 85 \\
ComunBaB & 1.68 & .790 & 85 \\
ExpdeUsuario & 2.36 & 1.153 & 85 \\
RetroClientes & 1.95 & .815 & 85 \\
SecDifMensa & 1.85 & .809 & 85 \\
IdentExtranje & 2.07 & .799 & 85
\end{tabular}


OpinSinInters

2.11

.787

85

Fuente. Elaboración propia

Tabla 7. Descriptivos de ítems del constructo Calidad de las publicaciones

\begin{tabular}{lccc}
\hline Estadísticas de elemento & Media & Desviación estándar & $\mathrm{N}$ \\
\hline ServPostPub & 3.16 & 1.421 & 85 \\
ConfiabPublic & 2.34 & 1.007 & 85 \\
LealtCliente & 2.55 & .982 & 85 \\
SimetriaClient & 2.53 & .946 & 85 \\
InfRelevProd & 2.41 & .955 & 85 \\
MejorCompañ & 2.39 & 1.013 & 85 \\
ExpresSatisf & 2.19 & 1.018 & 85 \\
DatosProduct & 2.26 & 1.014 & 85 \\
IncrComprCali & 2.04 & .944 & 85 \\
\hline
\end{tabular}

Fuente. Elaboración propia 